\title{
MATRIČNE FAKTORIZACIJE
}

\author{
MATRIX DECOMPOSITION
}

\author{
Ines Radošević Medvidović* , Kristina Pedić
}

\begin{abstract}
Sažetak
Matrice se dijele u različite klase, ovisno o formi i određenim svojstvima. Matrične faktorizacije ovise o svojstvima određene klase matrica pa su faktorizacije matrica od velikog značaja u teoriji matrica, pri analizi numeričkih algoritama i uopće u numeričkoj linearnoj algebri. Faktorizacija matrice $A$ je prikaz matrice A kao produkta "jednostavnijih" matrica, što omogućuje jednostavnije rješavanje nekog problema. U teoriji matrica značajne su faktorizacije onih matrica kod kojih je moguća transformacija sličnost, kod što su Schurova dekompozicija, spektralna dekompozicija, singularna dekompozicija. Nadalje, osnovni alat za rješavanje sustava linearnih jednadžbi, kao jednog od osnovnih problema numeričke linearne algebre, je $L U$ faktorizacija. Također, bitno je spomenuti i $Q R$ faktorizaciju i njeno računanje preko rotacija i reflektora.
\end{abstract}

Ključne riječi: numerička analiza, teorija matrica, Jordanova forma, Schurova dekompozicija, LU faktorizacija, QR faktorizacija, singularna dekompozicija

\begin{abstract}
Matrices are divided into different classes, depending on the form and specific properties of the matrix. Matrix factorizations depend on the properties of certain class of matrices, hence matrix factorization are of great importance in the matrix theory, in the analysis of numerical algorithms and even in numerical linear algebra. $A$ factorization of the matrix $A$ is a representation of $A$ as a product of several "simpler" matrices, which makes the problem at hand easier to solve. Factorizations of matrices into some special sorts of matrices with similarity are of fundamental importance in matrix theory, like Schur decomposition, spectral decomposition and the singular value decomposition. Furthermore, the basic tool for solving systems of linear equations, as one of the basic problems of numerical linear algebra, is the LU factorization. Also, it is important to mention $Q R$ factorization and its calculation through rotation and reflectors.
\end{abstract}

\footnotetext{
* Odjel za matematiku, Sveučilište u Rijeci, Radmile Matejčić 2, Rijeka, Hrvatska E-mail: inesr@math.uniri.hr; kristina.pedic@student.uniri.hr
} 
Key words: numerical analysis, matrix theory, Jordan canonical form, Schur decomposition, $L U$ decomposition, $Q R$ decomposition, singular value decomposition

\section{Uvod}

Matrična faktorizacija daje prikaz matrice kao produkt matrica, pri čemu odabir faktorizacije ovisi o svojstvu faktorizirane matrice. Dvije su osnovne grupe matričnih faktorizacija, one povezane s rješavanjem sustava linearnih jednadžbi i one povezane s problemom vlastitih vrijednosti. Ovdje će biti opisane neke od njih. Ovaj rad ne sadrži rezultate numeričke linearne algebre korištene u obradi teme, niti su dane definicije i tvrdnje iz teorije matrica, već se smatra da su čitaču poznate. Samo neki pojmovi iz teorije matrica koji se koriste su: trokutasta matrica, dijagonalna matrica, hermitska (simetrična) matrica, unitarna (ortogonalna) matrica, pozitivno definitna matrica, normalna matrica, te pojam vektorske i matrične norme, ranga matrice vidi [8] [11] [19]. Još neki pojmovi koji se koriste i potrebno ih je poznavati za razumijevanje teme su pojam vektora, vektorskog prostora, ortogonalnosti, problem vlastitih vrijednosti i vlastitih vektora (svojstvenih vrijednosti i svojstvenih vektora), algebarske i geometrijske višestrukosti vlastitih vrijednosti, linearnog operatora i matričnog zapisa linearnog operatora, pojam linearne zavisnosti vektora i vezu $s$ dijagonalizibilnosti matrice, [9] [10]. Linearni operator $f: V \rightarrow V$, na vektorskom prostoru $\mathrm{V}$, dopušta dijagonalizaciju ako postoji baza u kojoj je operator reprezentiran dijagonalnom matricom. Dijagonalizibilnost matrice $A$ vezana je za pojam sličnosti, odnosno matrični zapis $A$ operatora $f$ u nekoj bazi vektorskog prostora $\mathrm{V}$. Dijagonalizibilna matrica dopušta rastav $A=S \Lambda S^{-1}$, pri čemu je $S$ koordinatna matrica vlastitih vektora operatora $f$ u istoj bazi u kojoj je $A$ njegov matrični zapis, $S^{-1}$ je inverzna matrica od $S$, a $\Lambda$ je dijagonalna matrica s vlastitim vrijednostima tog operatora na dijagonali. Naime, dvije kvadratne matrice istog reda $n$, kao ovdje $A$ i $\Lambda$, su slične ako je $A=S \Lambda S^{-1}$ za invertibilnu matricu $S$ reda $n$. Ako je matrica $S$ unitarna, onda je matrica $A$ unitarno dijagonalizibilna (za realnu matricu $A$ kaže se da je ortogonalno dijagonalizibina). Nadalje, matrice koje nemaju pun sustav vlastitih vektora ne mogu se dijagonalizirati pomoću transformacije sličnosti. Ovo su smjernice za neke matrične faktorizacije koje su vezana za određena svojstva matrica koje želimo faktorizirati.

\section{Matrične faktorizacije}

\subsection{Jordanova forma}

Kvadratnu matricu, koja se ne može dijagonalizirati ni nad kojim poljem, pokušava se svesti na oblik koji je onoliko blizak dijagonalnom obliku koliko 
matrica dopušta, vidi [8] [10]. Takvu matricu se pokuša kvazidijagonalizirati, odnosno svesti na dijagonalnu blok matricu, sa što je moguće jednostavnijom strukturom blokova. To vodi na pojam Jordanove ${ }^{1}$ forme matrice ili Jordanovog normalnog oblika matrice. Elementarna Jordanova klijetka (blok), pridružena skalaru $\lambda$ je kvadratna matrica oblika

$$
J_{k}(\lambda)=\left[\begin{array}{cccc}
\lambda & 1 & & \\
& \lambda & \ddots & \\
& & \ddots & 1 \\
& & & \lambda
\end{array}\right] \in \mathbb{C}^{k \times k} .
$$

Jordanova klijetka (blok) je dijagonalna blok matrica oblika

$$
J(\lambda)=\left[\begin{array}{llll}
J_{k_{1}}(\lambda) & & & \\
& J_{k_{2}}(\lambda) & & \\
& & \ddots & \\
& & & J_{k_{r}}(\lambda)
\end{array}\right] \in \mathbb{C}^{l \times l},
$$

pridružena skalaru $\lambda$, gdje su $J_{k_{r}}(\lambda)$ elementarne Jordanove klijetke, iz (1) i pri čemu je $k_{1} \geq k_{2} \geq \cdots \geq k_{r} \geq 1$ i $k_{1}+k_{2}+\cdots+k_{r}=l \leq n$. Jordanova matrica

$$
J=\left[\begin{array}{llll}
J\left(\lambda_{1}\right) & & & \\
& J\left(\lambda_{2}\right) & & \\
& & \ddots & \\
& & & J\left(\lambda_{s}\right)
\end{array}\right] \in \mathbb{C}^{n \times n},
$$

je dijagonalna blok matrica gdje su $J\left(\lambda_{j}\right)$ Jordanovi blokovi koji pripadaju različitim skalarima $\lambda_{j}$. Sljedeći teorem je jedan od osnovnih teorema koji daje kanonsku strukturu matrica, određuje Jordanovu formu, formu matrice najbližu dijagonalnoj za matrice koje se ne mogu dijagonalizirati.

Teorem 2.1.1. [9, str.126 -127] Za proizvoljnu matricu $A \in \mathbb{C}^{n \times n}$ postoji regularna matrica $S$ takva da je

$$
A=S\left[\begin{array}{llll}
J\left(\lambda_{1}\right) & & & \\
& J\left(\lambda_{2}\right) & & \\
& & \ddots & \\
& & & J\left(\lambda_{p}\right)
\end{array}\right] S^{-1}=S J S^{-1},
$$

gdje su $\boldsymbol{J}\left(\boldsymbol{\lambda}_{\boldsymbol{i}}\right), \boldsymbol{n}_{\boldsymbol{i}} \times \boldsymbol{n}_{\boldsymbol{i}}$ Jordanovi blokovi međusobno različitih vlastitih vrijednosti $\lambda_{1}, \ldots, \lambda_{p}$ od $\boldsymbol{A}, \mathrm{s}$ algebarskim kratnostima $\boldsymbol{n}_{1}, \ldots, \boldsymbol{n}_{p}$, respektivno, i pritom je $\sum_{\boldsymbol{i}=\mathbf{1}}^{p} \boldsymbol{n}_{\boldsymbol{i}}=\boldsymbol{n}$.

${ }^{1}$ Camille Jordan (1838. - 1922.), francuski matematičar. 
Stupci matrice $\boldsymbol{S}$ su vlastiti vektori, a vrijednosti $\lambda_{\boldsymbol{i}}$ za $\boldsymbol{i}=\mathbf{1}, \ldots, \boldsymbol{p}$ su vlastite vrijednosti matrice $\boldsymbol{A}$, pri čemu skup vlastitih vrijednosti od $\boldsymbol{A}$ čini spektar od $\boldsymbol{A}$. Općenito vijedi da za svaki $\boldsymbol{i}$ postoji vektor $\boldsymbol{v}_{\boldsymbol{i}} \neq \mathbf{0}$ takav da je $\boldsymbol{A} \boldsymbol{v}_{\boldsymbol{i}}=$ $\lambda_{i} \boldsymbol{v}_{\boldsymbol{i}}$. Vektor $\boldsymbol{v}_{\boldsymbol{i}}$ naziva se vlastiti vektor matrice $A$, koji je pridružen vlastitoj vrijednosti $\lambda_{i}$, a broj Jordanovih blokova $\boldsymbol{p}$ predstavlja broj nezavisnih vlastitih vektora matrice $\boldsymbol{A}$. Matrica $\boldsymbol{A}$ je dijagonalizibilna ako i samo ako je $\boldsymbol{p}=\boldsymbol{n}$, i onda svi stupci matrice $\boldsymbol{S}$ predstavljaju vlastite vektore, a matrica $\boldsymbol{J}$ je dijagonalna jer su joj samo dijagonalni elementi eventualno različiti od nule.

Jordanova matrica $\boldsymbol{J}$ jedinstvena je do permutacije u njezinim dijagonalnim blokovima. Za realnu matricu $\boldsymbol{A}$ s realnim vlastitim vrijednostima, matrica $\boldsymbol{S}$ je realna. Jordanov blok $\boldsymbol{J}\left(\boldsymbol{\lambda}_{\mathbf{1}}\right)$ je oblika kao u (2) pa za svako $\mathbf{1} \leq \boldsymbol{i} \leq \boldsymbol{p}$, postoji rastav $\boldsymbol{n}_{\boldsymbol{i}}=\boldsymbol{m}_{\boldsymbol{i}, \mathbf{1}}+\cdots+\boldsymbol{m}_{\boldsymbol{i}, \boldsymbol{r}_{\boldsymbol{i}}}$, pri čemu je $\boldsymbol{m}_{\boldsymbol{i}, \boldsymbol{j}}$ dimenzija j-tog po redu elementarnog Jordanovog bloka u $\boldsymbol{J}\left(\boldsymbol{\lambda}_{\boldsymbol{i}}\right)$. Za $\boldsymbol{p}=\mathbf{1}, \boldsymbol{r}_{\mathbf{1}}=\mathbf{1}$ Jordanov oblik od $\boldsymbol{A}$ je jedan elementaran Jordanov blok reda $\boldsymbol{n}$. Za $\boldsymbol{p}=\boldsymbol{n}, \boldsymbol{n}_{\boldsymbol{i}}=\mathbf{1}$ za svaki $\boldsymbol{i}$ (pa je $\boldsymbol{r}_{\boldsymbol{i}}=\mathbf{1}$ i $\boldsymbol{m}_{\boldsymbol{i}, \mathbf{1}}=\mathbf{1}$ ), što znači da je $\boldsymbol{J}\left(\boldsymbol{\lambda}_{\boldsymbol{i}}\right)$ jedan elementarni Jordanov blok. Ovo vodi na zaključak, da je matrica dijagonalizibilna ako su Jordanovi blokovi u Jordanovoj formi matrice reda 1, a ako postoji barem jedan blok reda barem dva, $\boldsymbol{A}$ je defektna matrica. Svojstvo defektnih matrica je da nemaju punu bazu vlastitih vektora, o čemu govori geometrijska kratnost vlastite vrijednosti.

\subsection{Schurova dekompozicija}

Jedan od osnovnih alata u analiziranju i numeričkom rješavanju problema vlastitih vrijednosti je Schurova dekompozicija. Upravo nam ova dekompozicija daje odgovor na pitanje koliko najviše možemo unitarnom transformacijom sličnosti pojednostaviti matricu $\boldsymbol{A} \in \mathbb{C}^{\boldsymbol{n} \times \boldsymbol{n}}$. Vlastite vrijednosti i vlastiti vektori matrice $\boldsymbol{A}$ jednostavno se prenose na njoj sličnu matricu $\boldsymbol{B}=\boldsymbol{S}^{-1} \boldsymbol{A S}$, ako je $\boldsymbol{B} \boldsymbol{x}=\boldsymbol{\lambda} \boldsymbol{x}$, onda je $\boldsymbol{A S} \boldsymbol{x}=\boldsymbol{\lambda} \boldsymbol{S} \boldsymbol{x}$. Dakle, za računanje vlastitih vrijedosti i vlastitih vektora matrice $\boldsymbol{A}$ praktično je tražiti transformacije sličnosti koje će dati matricu $\boldsymbol{B}$ koja je jednostavnije spektralne strukture. Unitarna (ortogonalna) transformacija sličnosti $\boldsymbol{S}$ ima prednost zbog nekih teoretskih i numeričkih svojstava, kao što je jednostavnije računanje inverza matrice transformacije, budući vrijedi $\boldsymbol{S}^{-\mathbf{1}}=\boldsymbol{S}^{*}$ te ne povećava normu perturbacije, čuva neke klase matrica. Pa ako je $\boldsymbol{A}$ normalna, hermitska, antihermitska, unitarna, onda je matrica $\boldsymbol{B}$ 
$\left(\boldsymbol{B}=\boldsymbol{S}^{*} \boldsymbol{A} \boldsymbol{S}\right)$ normalna, hermitska, antihermitska, unitarna, respektivno. Unitarna transformacija sličnosti matrice $\boldsymbol{A}$ dana je u sljedećem teoremu.

Teorem 2.2.1. [9, str.79-80] (Schurova ${ }^{2}$ dekompozicija) Neka je $\boldsymbol{A} \in \mathbb{C}^{\boldsymbol{n} \times \boldsymbol{n}}$ i neka su $\lambda_{1}, \ldots, \lambda_{n}$ njene vlastite vrijednosti u proizvoljnom poretku. Postoji unitarna matrica $\boldsymbol{U}$ i gornjetrokutasta matrica $\boldsymbol{T}$ tako da je $\boldsymbol{U}^{*} \boldsymbol{A U}=\boldsymbol{T}=$ $\left[\boldsymbol{t}_{\boldsymbol{i} j}\right]$, pri čemu su dijagonalni elementi $\boldsymbol{t}_{\boldsymbol{i} i}=\boldsymbol{\lambda}_{\boldsymbol{i}}, \boldsymbol{i}=\mathbf{1}, \ldots, \boldsymbol{n}$. Ako je $\boldsymbol{A} \in \mathbb{R}^{\boldsymbol{n} \times \boldsymbol{n}}$ i ako su sve vlastite vrijednosti od $\boldsymbol{A}$ realne, onda je $\boldsymbol{T}$ također realna i može se odabrati $U$ kao realna ortogonalna. Zapis $\boldsymbol{A}=\boldsymbol{U} \boldsymbol{T} \boldsymbol{U}^{*}$ se naziva Schurova dekompozicija matrice $\boldsymbol{A}$, a matrica $\boldsymbol{T}$ je Schurova forma od $\boldsymbol{A}$.

Prema teoremu 2.2.1., svaka kvadratna matrica $\boldsymbol{A}$ je unitarno slična trokutastoj matrici, $\boldsymbol{A}=\boldsymbol{U} \boldsymbol{T} \boldsymbol{U}^{*}$, čiji su dijagonalni elementi vlastite vrijednosti matrice $\boldsymbol{A}$ u nekom proizvoljnom poretku. Kako slične matrice imaju isti trag, determinantu te svojstvene vrijednosti, Schurov teorem predstavlja vrlo koristan rezultat u teoriji matrica. U dokazu teorema 2.2.1. na matricu $\boldsymbol{A}$ se djeluje unitarnim transformacijama sličnosti sve dok ne postane trokutasta. U tom postupku se direktno koriste vlastite vrijednosti i vlastiti vektori, što zapravo nije praktično jer u primjeni nisu dostupni i nije ih lako izračunati. Numeričko računanje Schurove dekompozicije se svodi na beskonačan niz transformacija sličnosti koje reduciraju elemente ispod glavne dijagonale i osiguravaju trokutastu formu tek u limesu. Schurova forma nije jedinstvena jer se vlastite vrijednosti polazne matrice mogu pojaviti na dijagonali matrice $\boldsymbol{T}$ u bilo kojem poretku.

Primjer 2.2.1. Schurova dekompozicija $2 \times 2$ matrice $A=\left[\begin{array}{cc}5 & 7 \\ -2 & -4\end{array}\right]$. Treba pronaći ortogonalnu matricu $U$ i gornjetrokutastu matricu $T$ tako da je $U^{T} A U=T$, primjenom dokaza teorema Schurove dekompozicije. Iz $\operatorname{det}(A-\lambda I)=0 \Leftrightarrow \lambda^{2}-\lambda-6=0$ slijedi $\lambda_{1}=-2$ i $\lambda_{2}=3$. Za vlastitu vrijednost $\lambda_{1}=-2$ slijedi

$$
(A+2 I) X_{1}=0 \Leftrightarrow\left[\begin{array}{cc}
7 & 7 \\
-2 & -2
\end{array}\right]\left[\begin{array}{l}
x_{1} \\
x_{2}
\end{array}\right]=\left[\begin{array}{l}
0 \\
0
\end{array}\right] \Rightarrow X_{1}=(1,-1) .
$$

Za vlastitu vrijednost $\lambda_{2}=3$ slijedi

$$
(A-3 I) X_{2}=0 \Leftrightarrow\left[\begin{array}{cc}
2 & 7 \\
-2 & -7
\end{array}\right]\left[\begin{array}{l}
x_{1} \\
x_{2}
\end{array}\right]=\left[\begin{array}{l}
0 \\
0
\end{array}\right] \Rightarrow X_{2}=(7,-2) .
$$

Ortonormiranjem vektora $X_{1}=(1,-1)$ i $X_{2}=(7,-2)$ dobiva se ortonormirani skup $\left\{\left(\frac{1}{\sqrt{2}}, \frac{-1}{\sqrt{2}}\right),\left(\frac{1}{\sqrt{2}}, \frac{1}{\sqrt{2}}\right)\right\}$. Slijedi $U=\frac{1}{\sqrt{2}}\left[\begin{array}{cc}1 & 1 \\ -1 & 1\end{array}\right]$. Konačno, Schurova dekompozicija matrice $A$ je

$$
T=U^{T} A U=\left[\begin{array}{cc}
-2 & 9 \\
0 & 3
\end{array}\right]
$$

${ }^{2}$ Issai Schur (1875.-1941.), njemački matematičar. 
Matrice koje možda imaju i višestruke vlastite vrijednosti, a mogu se dijagonalizirati, su normalne matrice. Schurov teorem ima neke važne posljedice, kao što je sljedeći korolar koji kaže da je Schurova dekompozicija normalnih matrica dijagonalna. Unitarne i hermitske matrice su klase normalnih matrica pa se sljedeći teorem odnosi i na njih. Unitarno slična matrica normalnoj matrici je opet normalna; za normalnu matricu $A$ i $U^{*} A U=T$ Schurovu formu, gdje je $U$ neka unitarna matrica, vrijedi

$$
\boldsymbol{T}^{*} \boldsymbol{T}=\boldsymbol{U}^{*} \boldsymbol{A}^{*} \boldsymbol{U} \boldsymbol{U}^{*} \boldsymbol{A} \boldsymbol{U}=\boldsymbol{U}^{*} \boldsymbol{A}^{*} \boldsymbol{A} \boldsymbol{U}=\boldsymbol{U}^{*} \boldsymbol{A} \boldsymbol{A}^{*} \boldsymbol{U}=\boldsymbol{U}^{*} \boldsymbol{A} \boldsymbol{U} \boldsymbol{U}^{*} \boldsymbol{A}^{*} \boldsymbol{U}=\boldsymbol{T} \boldsymbol{T}^{*},
$$

pa je $\boldsymbol{T}$ normalna matrica. Za hermitsku matricu $\boldsymbol{A}$ i $\boldsymbol{U}^{*} \boldsymbol{A} \boldsymbol{U}=\boldsymbol{T}$ Schurovu formu, gdje je $\boldsymbol{U}$ neka unitarna matrica, vrijedi

$$
\boldsymbol{T}^{*}=\left(\boldsymbol{U}^{*} \boldsymbol{A U}\right)^{*}=\boldsymbol{U}^{*} \boldsymbol{A}^{*} \boldsymbol{U}=\boldsymbol{U}^{*} \boldsymbol{A} \boldsymbol{U}=\boldsymbol{T},
$$

pa je T hermitska matrica. Kako je $\boldsymbol{T}$ gornjetrokutasta matrica, slijedi da je $\boldsymbol{T}^{*}$ donjetrokutasta matrica, pa je $\boldsymbol{T}$ dijagonalna.

Korolar 2.2.2. Trokutasta matrica $\boldsymbol{T}$ u Schurovoj dekompoziciji $\boldsymbol{A}=\boldsymbol{U} \boldsymbol{T} \boldsymbol{U}^{*}$ je dijagonalna ako i samo ako je matrica $\boldsymbol{A} \in \mathbb{C}^{\boldsymbol{n} \times \boldsymbol{n}}$ normalna, što je također ekvivalentno sa $\|A\|_{F}^{2}=\sum_{i=1}^{n}\left|\lambda_{i}(A)\right|^{2}$.

Spektralni teoremi slijede iz već spomenute činjenice da Schurova forma, osim što je trokutasta, nasljeđuje svojstva hermitičnosti, antihermitičnosti i unitarnosti. Dakle, normalna matrica A je oblika

$$
\boldsymbol{A}=\boldsymbol{U} \boldsymbol{\Lambda} \boldsymbol{U}^{*},
$$

gdje je $\boldsymbol{\Lambda}$ dijagonalna matrica, a $\boldsymbol{U}$ unitarna. Zapis matrice $\boldsymbol{A}$ u obliku danom u (3), gdje je $\boldsymbol{U}=\left[\boldsymbol{u}_{1}, \ldots, \boldsymbol{u}_{\boldsymbol{n}}\right]$ unitarna matrica čiji su stupci vlastiti vektori od $\boldsymbol{A}$, a $\boldsymbol{\Lambda}$ dijagonalna matrica s realnim vlastitim vrijednostima od $\boldsymbol{A}$ na glavnoj dijagonali, zove se spektralna dekompozicija ${ }^{3}$ od $\boldsymbol{A}$. Mogu se izreći sljedeći spektralni teoremi [9, str.101-104]:

Schurova forma hermitske matrice je realna dijagonalna matrica (vrijedi $\left.\bar{\lambda}_{i}=\lambda_{i}, \boldsymbol{i}=1, \ldots, n\right)$. Schurova forma antihermitske matrice je dijagonalna matrica s čisto imaginarnim dijagonalnim elementima (vrijedi $\bar{\lambda}_{i}=-\lambda_{i}$, $\boldsymbol{i}=\mathbf{1}, \ldots, \boldsymbol{n})$. Schurova forma unitarne matrice je dijagonalna matrica s $\left|\lambda_{j}\right|=\mathbf{1}, \boldsymbol{j}=\mathbf{1}, \ldots, \boldsymbol{n}$ (vlastite vrijednosti se nalaze na jediničnoj kružnici).

Za vlastite vrijednosti $\lambda_{\boldsymbol{i}}$ pozitivno definitne matrice vrijedi $\boldsymbol{u}_{\boldsymbol{i}}^{*} \boldsymbol{A} \boldsymbol{u}_{\boldsymbol{i}}=$ $\left(\boldsymbol{A} \boldsymbol{u}_{i}, \boldsymbol{u}_{i}\right)=\left(\boldsymbol{\lambda}_{\boldsymbol{i}} \boldsymbol{u}_{i}, \boldsymbol{u}_{i}\right)=\boldsymbol{\lambda}_{\boldsymbol{i}}\left\|\boldsymbol{u}_{\boldsymbol{i}}\right\|_{2}^{2}>\mathbf{0}$, gdje je $\boldsymbol{u}_{\boldsymbol{i}}$ vlastiti vektor različit od nul-vektora, $\left\|\boldsymbol{u}_{i}\right\|_{2}>\mathbf{0}$, pa mora biti $\lambda_{\boldsymbol{i}}>\mathbf{0}$ za $\boldsymbol{i}=\mathbf{1}, \ldots, \boldsymbol{n}$. Prema tome,

${ }^{3}$ EVD je akronim za Eigenvalue decomposition (engl.). 
vlastite vrijednosti pozitivno definitne matrice su pozitivne. Analogno, vlastite vrijednosti pozitivno semidefinitne matrice su sve veće ili jednake nuli. Iz svojstva matričnog množenja, za zapis iz (3), često se koristi ekvivalentan zapis

$$
\boldsymbol{A}=\sum_{i} \lambda_{\boldsymbol{i}} \boldsymbol{u}_{\boldsymbol{i}} \boldsymbol{u}_{\boldsymbol{i}}^{*}
$$

Zapis iz (4) spektralne dekompozicije koristan je pri računanju inverza, ili općenito $n$-te potencije matrice $\boldsymbol{A}$, pa je $\boldsymbol{A}^{-\mathbf{1}}=\boldsymbol{U} \boldsymbol{\Lambda}^{-1} \boldsymbol{U}^{*}=\sum_{\boldsymbol{i}} \frac{\mathbf{1}}{\lambda_{\boldsymbol{i}}} \boldsymbol{u}_{\boldsymbol{i}} \boldsymbol{u}_{\boldsymbol{i}}^{*}$ i općenito $\boldsymbol{A}^{\boldsymbol{n}}=\boldsymbol{U} \boldsymbol{\Lambda}^{n} \boldsymbol{U}^{*}$. Realna hermitska matrica naziva se simetrična matrica i može se dijagonalizirati pomoću transformacije sličnosti $\mathrm{s}$ ortogonalnom matricom pa se njena spektralna dekompozicija zapisuje u obliku $\boldsymbol{A}=\boldsymbol{Q} \boldsymbol{\Lambda} \boldsymbol{Q}^{\boldsymbol{T}}$, gdje je $\boldsymbol{\Lambda}$ dijagonalna matrica vlastitih vrijednosti od $\boldsymbol{A}$, a $\boldsymbol{Q}$ ortogonalna matrica čiji su stupci vlastiti vektori od $\boldsymbol{A}$.

\subsection{LU faktorizacija}

Rješavanje linearnih sustava jednadžbi $\boldsymbol{A} \boldsymbol{x}=\boldsymbol{b}$ jedan je od osnovnih problema numeričke linearne algebre. Matrica $A=\left[a_{i j}\right]_{i, j=1}^{n} \in \mathbb{R}^{n \times n}$ je matrica sustava, a njeni elementi su koeficijenti uz nepoznanice, vektor $\boldsymbol{x}=$ $\left[x_{i}\right]_{i=1}^{n} \in \mathbb{R}^{n}$ je vektor nepoznanica i vektor $\boldsymbol{b}=\left[\boldsymbol{b}_{\boldsymbol{i}}\right]_{i=\mathbf{1}}^{n} \in \mathbb{R}^{\boldsymbol{n}}$ je vektor desne strane sustava, elementi su mu slobodni koeficijenti. Rješenje sustava dobiva se množenjem jednadžbe $\boldsymbol{A x}=\boldsymbol{b}$ s lijeve strane matricom $\boldsymbol{A}^{-\mathbf{1}}$, $A^{-1} A x=A^{-1} b \Rightarrow x=A^{-1} b$. Takav način rješavanja ne koristi se često jer dovodi do problema traženja inverza i množenja matrica i vektora. Najpoznatiji i najjednostavniji algoritam za rješavanje sustava linearnih jednadžbi je svođenje sustava na njemu ekvivalentan sustav koristeći Gaussovu 4 metodu eliminacije, vidi [5] [8]. Proces eliminacije interpretira se kao faktorizacija matrice sustava $\boldsymbol{A}$ na produkt trokutastih matrica. Trokutasta faktorizacija iz sljedećeg teorema osnova je spomenute metode.

Teorem 2.3.1. [8, str.150] (LU faktorizacija). Neka je matrica $A \in \mathbb{C}^{n \times n}$. Onda postoji matrica permutacije $P$ takva da Gaussove eliminacije daju $L U$ faktorizaciju

$$
P A=L U,
$$

matrice $P A$, pri čemu je matrica $L$ donjetrokutasta $\mathrm{s}$ jedinicama na dijagonali, a $U$ gornjetrokutasta matrica.

Faktorizacija oblika kao u (5) je LU faktorizacija s parcijalnim pivotiranjem, gdje je $P$ matrica permutacije ili permutacija koja permutira retke matrice

\footnotetext{
${ }^{4}$ Johann Carl Friedrich Gauß, lat. Carolus Fridericus Gauss, (1777.-1855.), njemački matematičar.
} 
$A$ da bi se osigurala faktorizacija. Naime, izvedivost Gaussovih eliminacija koje dovode do faktorizacije ovisi o uvjetu da su pivotni elementi različiti od nule, što je vidljivo iz algoritma 2.3.2., a ti uvjeti su osigurani ako su matrici $A$ determinante glavnih podmatrica $A(1: k, 1: k), k=1, \ldots, n-1$, različite od nule. Ako je $A=L U$ faktorizacija matrice $A$, pri čemu je matrica $L$ donjetrokutasta s jedinicama na dijagonali, a $U$ gornjetrokutasta matrica, onda se sustava jednadžbi $A x=L U x=b$ rješava upravo kao dva jednostavna linearna sustava. Neka je $L y=b$ donjetrokutasti sustav i $U x=y$ gornjetrokutasti sustav. Faktorizacija matrice $A$ označava se sa $P A$ kao u (5), gdje je $P$ matrica permutacije koja u svakom retku i svakom stupcu ima točno jednu jedinicu. Na samom početku ona je jednaka jediničnoj matrici. Dakle, sustav $A x=b$ se rješava pomoću $L U$ faktorizacije tako da se pomnoži s maticom permutacije $P$ čime se dobiva $P A x=L U x=$ $P b$. Vektor $b^{\prime}=P b$ je vektor koji ima permutirane komponente vektora $b$. Postupak se nastavlja s $L U x=b^{\prime}$.

Primjer 2.3.1. Rješenje sustava linearnih jednadžbi Gaussovom metodom s parcijalnim pivotiranjem

$$
\begin{array}{cccc}
x_{1}+x_{2} & +x_{3} & =4 \\
x_{1} & +2 x_{2} & +3 x_{3} & =5 \\
5 x_{1} & +3 x_{2} & +5 x_{3} & =14
\end{array}
$$

Najveći element u prvom stupcu matrice sustave $A=\left[\begin{array}{lll}1 & 1 & 1 \\ 1 & 2 & 3 \\ 5 & 3 & 5\end{array}\right]$ je na poziciji $(3,1)$ pa zamjenom prvog i trećeg redka maksimizira se prvi pivotni element. Zamjena redaka u matrici biti će provedana djelovanjem matrice permutacija $P^{(1)}=\left[\begin{array}{lll}0 & 0 & 1 \\ 0 & 1 & 0 \\ 1 & 0 & 0\end{array}\right], P^{(1)} A=\left[\begin{array}{lll}5 & 3 & 5 \\ 1 & 2 & 3 \\ 1 & 1 & 1\end{array}\right]$. Sada se definira $L^{(1)}=$ $\left[\begin{array}{ccc}1 & 0 & 0 \\ -\frac{1}{5} & 1 & 0 \\ -\frac{1}{5} & 0 & 1\end{array}\right]$, pa je $A^{(1)}=L^{(1)} P^{(1)} A=\left[\begin{array}{ccc}5 & 3 & 5 \\ 0 & \frac{7}{5} & 2 \\ 0 & \frac{2}{5} & 0\end{array}\right]$. Sljedeći pivotni element je maksimiziran identičnom transformacijom $P^{(1)}=I$. Definira se $L^{(2)}=$ $\left[\begin{array}{ccc}1 & 0 & 0 \\ 0 & 1 & 0 \\ 0 & -\frac{2}{7} & 1\end{array}\right]$, pa je $A^{(2)}=L^{(2)} P^{(2)} A^{(1)}=L^{(2)} I A^{(1)}=\left[\begin{array}{ccc}5 & 3 & 5 \\ 0 & \frac{7}{5} & 2 \\ 0 & 0 & -\frac{4}{7}\end{array}\right]$. 
Sada slijedi $U \equiv A^{(2)}=L^{(2)} P^{(2)} L^{(1)} P^{(1)} A=L^{(2)} I L^{(1)} P^{(1)} A$. Dakle, $P^{(1)} A=$ $P A=\left[\begin{array}{lll}0 & 0 & 1 \\ 0 & 1 & 0 \\ 1 & 0 & 0\end{array}\right]\left[\begin{array}{lll}1 & 1 & 1 \\ 1 & 2 & 3 \\ 5 & 3 & 5\end{array}\right]=\left[\begin{array}{ccc}5 & 3 & 5 \\ 1 & 2 & 3 \\ 1 & 1 & 1\end{array}\right]=L^{(1)^{-1}} L^{(2)^{-1}} U$. Dobivena je faktorizacija $P A=L U=\left[\begin{array}{ccc}1 & 0 & 0 \\ \frac{1}{5} & 1 & 0 \\ \frac{1}{5} & 0 & 1\end{array}\right]\left[\begin{array}{lll}1 & 0 & 0 \\ 0 & 1 & 0 \\ 0 & \frac{2}{7} & 1\end{array}\right] U=\left[\begin{array}{ccc}1 & 0 & 0 \\ \frac{1}{5} & 1 & 0 \\ \frac{1}{5} & \frac{2}{7} & 1\end{array}\right]\left[\begin{array}{ccc}5 & 3 & 5 \\ 0 & \frac{7}{5} & 2 \\ 0 & 0 & -\frac{4}{7}\end{array}\right]$

matrice $P A$ na dvije trokutaste matrice. Iz matrične jednadžbe $P A x=$ $L U x=P b$ i s maticom permutacije $P=P^{(2)} P^{(1)}=\left[\begin{array}{lll}0 & 0 & 1 \\ 0 & 1 & 0 \\ 1 & 0 & 0\end{array}\right]$ dobiva se vektor $b^{\prime}=P b=\left[\begin{array}{lll}0 & 0 & 1 \\ 0 & 1 & 0 \\ 1 & 0 & 0\end{array}\right]\left[\begin{array}{c}4 \\ 5 \\ 14\end{array}\right]=\left[\begin{array}{c}14 \\ 5 \\ 4\end{array}\right]$. Sada zadani linearni sustav ima oblik $\left[\begin{array}{ccc}1 & 0 & 0 \\ \frac{1}{5} & 1 & 0 \\ \frac{1}{5} & \frac{2}{7} & 1\end{array}\right]\left[\begin{array}{ccc}5 & 3 & 5 \\ 0 & \frac{7}{5} & 2 \\ 0 & 0 & -\frac{4}{7}\end{array}\right]\left[\begin{array}{l}x_{1} \\ x_{2} \\ x_{3}\end{array}\right]=\left[\begin{array}{c}14 \\ 5 \\ 4\end{array}\right]$. Rješavanjem sustava $L y=b^{\prime}, \mathrm{s}$ donjetrokutastom matricom, dobiva se $\left[\begin{array}{l}y_{1} \\ y_{2} \\ y_{3}\end{array}\right]=\left[\begin{array}{l}14 \\ \frac{11}{5} \\ \frac{4}{7}\end{array}\right]$, te rješavanjem sustava $U x=y$, s gornjetrokutastom matricom, dobiva se $\left[\begin{array}{l}x_{1} \\ x_{2} \\ x_{3}\end{array}\right]=\left[\begin{array}{c}2 \\ 3 \\ -1\end{array}\right]$.

Za faktorizaciju nekih matrica, matrica $P$ nije potrebna pa se oblik iz relacije (5), gdje je $P$ sada identiteta, svodi na oblik $A=L U$. Primjer takvih matrica su pozitivno definitne matrice, kod kojih zbog hermitičnosti vrijedi da ako $L$ i $U^{*}$ imaju iste dijagonalne elemente, onda je $U=L^{*}$, pa LU faktorizacija pozitivno definitne matrice ima oblik $A=L L^{*}=U^{*} U$ koji se naziva faktorizacija Choleskog 5 ili trokutasta faktorizacija simetrične pozitivno definitne matrice, vidi [2, str. 46]. Ako faktorizacija $A=L U$ postoji i ako je matrica $A$ regularna, onda je faktorizacija jedinstvena, postoji točno jedna donjetrokutasta matrica $L \mathrm{~s}$ jedinicama na dijagonali i točno jedna gornjetrokutasta matrica $U$. Onda vrijedi i $\operatorname{det}(A)=\prod_{i=1}^{n} u_{i i}$.

${ }^{5}$ André-Louis Cholesky (1875.-1918.), francuski matematičar. 
Algoritam 2.3.2. [7, str.169] (Računanje LU faktorizacije matrice A)

$$
\begin{aligned}
& L=I ; \\
& z a k=1, \ldots, n-1 ; \\
& z a j=k+1, \ldots, n ; \\
& l_{j k}=\frac{a_{j k}^{(k-1)}}{a_{k k}^{(k-1)}} ; \\
& a_{j k}^{(k)}=0 ; \\
& z a j=k+1, \ldots, n ; \\
& z a i=k+1, \ldots, n ; \\
& a_{i j}^{(k)}=a_{i j}^{(k-1)}-l_{i k} a_{k j}^{(k-1)} ; \\
& U=A^{(n-1)}=\left[a_{i j}^{(n-1)}\right] .
\end{aligned}
$$

\subsection{QR faktorizacija}

Neka je zadan skup linearno nezavisnih vektora $a_{1}, a_{2}, \ldots, a_{n} \in \mathbb{R}^{m}\left(\mathbb{C}^{m}\right)$, smještenih u stupce matrice $A$ dimenzije $m \times n$. Za linearno nezavisan skup vektora nužno je $m \geq n$. Slijedi teorem koji daje još jednu metodu za rješavanje linearnih sustava i problema najmanjih kvadrata, vidi [6] [8].

Teorem 2.4.1. [9, str.112] (QR faktorizacija) Neka je $A \in \mathbb{C}^{m \times n}, m \geq n$. Onda postoji matrica $Q \in \mathbb{C}^{m \times n}$, s ortonormiranim stupcima i gornjetrokutasta matrica $R \in \mathbb{C}^{n \times n}$ tako da je

$$
A=Q R \text {. }
$$

Svaka matrica $A \in \mathbb{C}^{m \times n}\left(A \in \mathbb{R}^{m \times n}\right), m \geq n$, ima $Q R$ faktorizaciju, a ako je i punog ranga onda ima jedinstvenu $Q R$ faktorizaciju. Najjednostavniji dokaz ovog teorema je korištenjem Gram ${ }^{6}$-Schmidtovog7 postupka ortogonalizacije, vidi algoritam [8, str.218] [13]. Primjenom GramSchmidtovog postupka ortogonalizacije, vidi [1] [8], na stupce matrice $A$ dobivaju se jedinstvene matrice $Q$ i $R$, pri čemu matrica $Q$ ima ortonormirane stupce, a matrica $R$ je gornjetrokutasta $\mathrm{s}$ pozitivnim dijagonalnim elementima. Gram-Schmidtovim postupkom ortogonalizacije konstruira se ortonormirani skup vektora $q_{1}, q_{2}, \ldots, q_{n} \in \mathbb{R}^{m}\left(\mathbb{C}^{m}\right)$ koji razapinju isti potprostor $\mathrm{u} \mathbb{R}^{m}\left(\mathbb{C}^{m}\right)$. Neka je $r_{i j}=q_{i} \cdot a_{j}$ za $i \leq j$, oznaka za skalarni produkt vektora. Slijedi postupak u kojem se polazni stupac $a_{j}$ izražava kao linearna kombinaciju prvih $j$ vektora $q_{i}$ ortonormirane baze

\footnotetext{
${ }^{6}$ Jorgen Pedersen Gram (1850.-1916.), danski matematičar.

${ }^{7}$ Erhard Schmidt (1876.-1959.), njemački matematičar.
} 


$$
\begin{aligned}
& a_{1}=r_{11} q_{1}, \\
& a_{2}=r_{12} q_{1}+r_{22} q_{2}, \\
& a_{3}=r_{13} q_{1}+r_{23} q_{2}+r_{33} q_{3}, \\
& \cdots \\
& a_{n}=r_{1 n} q_{1}+r_{2 n} q_{2}+\cdots+r_{n n} q_{n} .
\end{aligned}
$$

Postupak se može zapisati u obliku umnoška matrica

$$
\left[\begin{array}{cccc}
a_{11} & a_{12} & \cdots & a_{1 n} \\
a_{21} & a_{22} & \cdots & a_{2 n} \\
\vdots & \vdots & \ddots & \vdots \\
a_{m 1} & a_{m 2} & \cdots & a_{m n}
\end{array}\right]=\left[\begin{array}{cccc}
q_{11} & q_{12} & \cdots & q_{1 n} \\
q_{21} & q_{22} & \cdots & q_{2 n} \\
\vdots & \vdots & \ddots & \vdots \\
q_{m 1} & q_{m 2} & \cdots & q_{m n}
\end{array}\right]\left[\begin{array}{cccc}
r_{11} & r_{12} & \cdots & r_{1 n} \\
0 & r_{22} & \cdots & r_{2 n} \\
\vdots & \vdots & \ddots & \vdots \\
0 & 0 & \cdots & r_{n n}
\end{array}\right]
$$

gdje je $\left(a_{k j}\right)_{k=1}^{m}=a_{j}$ i $\left(q_{k j}\right)_{k=1}^{m}=q_{j}$. Elementi $r_{i j}$ su elementi matrice $R$ i jasno je da se može uzeti $r_{i i}>0$. Ovim je dobivena faktorizacija matrice $A$ dimenzije $m \times n, m \geq n$, kao u teoremu 2.4.1., i naziva se reducirana $Q R$ faktorizacija matrice $A$. Može se izreći i druga varijanta iste faktorizacije, koja se dobiva iz reducirane i naziva se potpuna $Q R$ faktorizacija matrice $A$. Matrici $Q$ dodaje se $m-n$ dodatnih otronormiranih stupaca, tako da se dobije $\hat{Q} \in \mathbb{C}^{m \times m}$ unitarna matrica, odnosno ortogonalna u realnom slučaju, onda je $A=\hat{Q} \hat{R}$, gdje je $\hat{R} \in \mathbb{C}^{m \times n}$ matrica kojoj je gornji $n \times n$ blok jednak matrici $R$, a ostatak jednak nuli, $A=\hat{Q} \hat{R}=\left[\begin{array}{ll}Q & Q_{0}\end{array}\right]\left[\begin{array}{c}R \\ 0\end{array}\right]$.

Iako je ovaj dokaz ortogonalizacijom elegantan, u praksi se nikada ne koristi jer je nestabilan kad su stupci od $A$ skoro linearno zavisni. Umjesto toga, može se koristiti modificirani Gram-Schmidtov postupak ortogonalizacije, dan algoritmom u [8, str.218] [13], koji je stabilniji, ali i kod njega se može dogoditi da je izračunati $Q$ daleko od ortogonalne matrice.

Postoji više načina računanja $Q R$ faktorizacije, vidi [5], od kojih su najčešće korištena ona dva načina kod kojih se ortogonalna matrica $Q$ dobiva uzastopnim množenjem elementarnih ortogonalnih matrica. Dakle, primjenom niza unitarnih matrica na matricu $A$, kako bi se transformirala na gornjetrokutastu formu, dat će $Q R$ fakrtorizaciju matrice $A$, kao u (6). Budući da je produkt unitarnih matrica unitaran i inverz unitarne matrice također unitarna matrica. U tom postupku koriste se ili Givensove rotacije ili Householderovi reflektori kojima se poništavaju odgovarajući elementi u matrici $A$. Spomenuti postupci za računanje $Q R$ faktorizacije ovdje neće biti opisani, no lako ih je pronaći u literaturi iz numeričke analize, vidi [8] [13]. 


\subsection{Dekompozicija na singularne vrijednosti}

Singularna dekompozicija je temeljna dekompozicija u teoriji matrica i primjenama matrica u numeričkoj linearnoj algebra. Ima velike primjene u znanosti, tehnici, ekonomiji te u raznim praktičnim problemima, što je čini vjerojatno najvažnijom matričnom dekompozicijom. Dvije su grupe metoda za računanje singularne dekompozicije općih matrica. Metode koje se temelje na bidijagonalizaciji polazne matrice, kao što su QR, DQD (differential qd) i DC (divide i conquer) metode te metode koje se temelje na dijagonalizaciji polazne matrice, vidi [5], [18]. Računanje po bidijagonalizacijskim metodama je brže, ali je nezadovoljavajuće točnosti. Iz navedenog razloga sve je veći interes za dijagonalizacijskim metodama. Iterativne metode za računanje vlastitih vrijednosti i vlastitih vektora, vidi [14] te singularnih vrijednosti i vektora zadane matrice, temeljene na nizu ravninskih unitarnih transformacija sličnosti biranih tako da se izvandijagonalni dio matrice maksimalno reducira, nazivaju se Jacobijeve metode, vidi [8] [15], i pripadaju klasi dijagonalizacijskih metoda.

Faktorizacija matrice $A$ prikazana produktom $U \Sigma V^{*}$, gdje su $U$ i $V$ unitarne matrice, a $\Sigma$ dijagonalna matrica, naziva se singularna dekompozicija matrice $A$ ili kraće SVD $^{8}$ matrice $A$. SVD je temeljna dekompozicija u teoriji matrica i primjenama matrica u numeričkoj linearnoj algebri. Singularna dekompozicija matrice opisana je teoremom koji slijedi, čime su definirane singularne vrijednosti i singularni vektori matrice koja se promatra.

Teorem 2.5.1. [10, str. 144] (Singularna dekompozicija matrice) Ako je $A \in$ $\mathbb{C}^{m \times n}$, onda postoje unitarne matrice $U \in \mathbb{C}^{m \times m}$ i $V \in \mathbb{C}^{n \times n}$, takve da je

$$
U^{*} A V=\Sigma, \quad \Sigma=\operatorname{diag}\left(\sigma_{1}, \sigma_{2}, \ldots, \sigma_{\min \{m, n\}}\right),
$$

pri čemu vrijedi $\sigma_{1} \geq \sigma_{2} \geq \ldots \geq \sigma \min \{m, n\} \geq 0$.

Brojevi $\sigma_{1}, \sigma_{2}, \ldots, \sigma_{\min \{m, n\}}{ }^{9}$ su singularne vrijednosti matrice $A$. Stupci matrice $U$ su lijevi, a stupci matrice $V$ su desni singularni vektori matrice $A$.

Primjer 2.5.1. Singularna dekompozicija $3 \times 2$ matrice $A$ je (u Matlabu)

$$
A=\left[\begin{array}{ll}
1 & 2 \\
2 & 3 \\
3 & 4
\end{array}\right]=\left[\begin{array}{ccc}
-0.3381 & 0.8480 & 0.4082 \\
-0.5506 & 0.1735 & -0.8165 \\
-0.7632 & -0.5009 & 0.4082
\end{array}\right]\left[\begin{array}{cc}
6.5468 & 0 \\
0 & 0.3742 \\
0 & 0
\end{array}\right]\left[\begin{array}{cc}
-0.5696 & -0.8219 \\
-0.8219 & 0.5696
\end{array}\right]
$$

Singularne vrijednosti su unitarno invarijantne, jer ako je $A=$ $U \Sigma V^{*}$ singularna dekompozicija matrice $A$, onda je za proizvoljne unitarne

\footnotetext{
${ }^{8}$ SVD je akronim za Singular Value Decomposition (engl.).

${ }^{9}$ Matrica A ima $r(A)$ ne-nul singularnih vrijednosti i $\min \{m, n\}-r(A)$ nula singularnih vrijednosti
} 
matrice $W_{1} \in \mathbb{C}^{m \times m}$ i $W_{2} \in \mathbb{C}^{n \times n}, W_{1} A W_{2}=\left(W_{1} U\right) \Sigma$ pa je to singularna dekompozicija matrice $W_{1} A W_{2}$. Dakle, $A$ i $W_{1} C A$ matrice imaju iste singularne vrijednosti.

Može se pokazati da su singularne vrijednosti, $\sigma_{i}, 1 \leq i \leq \min \{m, n\}$, matrice $A$ kvadratni korijeni vlastitih vrijednosti matrica $A^{*} A$ i $A A^{*}$, [12]. Za $A \in$ $\mathbb{C}^{m \times n}$ obje matrice $A^{*} A$ i $A A^{*}$ su hermitske i pozitivno semidefinitne pa su njihove vlastite vrijednosti nenegativne.

Teorem 2.5.2. [4, str. 555] Neka je $A=U \Sigma V^{*}$ singularna dekompozicija matrice $A \in \mathbb{C}^{m \times n}$ ranga $r$. Onda je

(i) $V^{*}\left(A^{*} A\right) V=\operatorname{diag}(\sigma_{1}^{2}, \sigma_{2}^{2}, \ldots, \sigma_{r}^{2}, \underbrace{0, \ldots, 0}_{n-r})$;

pa su kvadrati singularnih vrijednosti matrice $A$ vlastite vrijednosti od $A^{*} A$ s dodatnih $n-r$ nula vlastitih vrijednosti. Stupci matrice $V$ su odgovarajući vlastiti vektori.

(ii) $U^{*}\left(A A^{*}\right) U=\operatorname{diag}(\sigma_{1}^{2}, \sigma_{2}^{2}, \ldots, \sigma_{r}^{2}, \underbrace{0, \ldots, 0}_{m-r})$;

pa su kvadrati singularnih vrijednosti matrıce $A$ viastite vrijednosti od $A A^{*}$ $\mathrm{s}$ dodatnih $m-r$ nula vlastitih vrijednosti. Stupci matrice $U$ su odgovarajući vlastiti vektori.

Prema definiciji singularnih vrijednosti i uzimajući za matricu $A$ normalnu matricu, može se zaključiti da su singularne vrijednosti normalne matrice apsolutne vrijednosti njezinih vlastitih vrijednosti.

Korolar 2.5.3. Neka je $A \in \mathbb{C}^{n \times n}$ normalna matrica s vlastitim vrijednostima $\lambda_{1}, \lambda_{2}, \ldots, \lambda_{n}$. Singularne vrijednosti od $A$ su $|\lambda i|, i=1, \ldots, n$.

SVD postoji za proizvoljne matrice i jedna je od najčešće korištenih alata za rješavanje niza problema u teoriji matrica, vidi [3], [10]. U singularnoj dekompoziciji pojavljuju se unitarne matrice, što je čini izvrsnim sredstvom za diskutiranje geometrije unitarnih prostora, pomoću nje se lako izračuna rang matrice te aproksimira matrica matricom manjeg ranga i jednostavno se izračunava generalizirani inverz matrice $A$. Moguće je proširiti pojam inverza matrice na matrice koje nisu regularne ili čak nisu niti kvadratne. Slijedi jedan od načina definiranja generaliziranog inverza.

SVD daje jednostavnu geometrijsku interpretaciju transformacije $x \mapsto$ $A x=U \Sigma V^{*} x$. Množenje unitarnim matricama $V^{*}$ i $U$ ne mijenja normu vektora, njihov geometrijski smisao je rotacija. Vektor $x$ se rotira u vektor $V^{*} x$, zatim se njegove komponente pomnože s nenegativnim brojevima $\sigma_{i}$, što znači da se mijenja njihova duljina, te se dobije vektor $\Sigma V^{*} x$. Na kraju vektor $\Sigma V^{*} x$ se rotira matricom $U$. Norma vektora $A x$ ovisi o singularnim vrijednostima matrice $A$. 


\subsection{Matrične faktorizacije u inženjerskim problemima}

Prikazane matrične faktorizacije u radu nalaze primjenu u svim granama tehnike, kao u tehničkoj mehanici, statici i dinamici konstrukcija, potresnom inženjerstvu, hidromehanici, mehanici fluida, ispitivanju materijala i konstrukcija, vidi [21]. U rješavanju inženjerskih problema često se pojavljuju sustavi linearnih jednadžbi $A x=b$. Konkretno, u svrhu ocjene stanja i ponašanja neke konstrukcije, konstrukcija se ispituje prema različitim shemama opterećenja, kao što je to dano primjerom rešetkastog nosača na Slici 1.

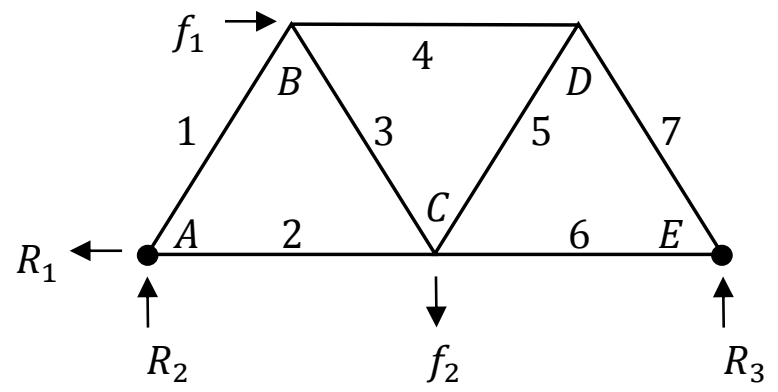

Slika 1. Shema opterećenja konstrukcije.

Na prikazanom primjeru čvorovi su označeni slovima $A, B, \ldots$, štapni elementi sa $1,2, \ldots$, reakcije oslonaca sa $R_{1}, R_{2}, R_{3}$, a sa $f_{1}$ i $f_{2}$ aplicirane sile u čvorovima za različite sheme opterećenja. Prikazana rešetka sastoji se od jednakostraničnih trokuta. Ukoliko na rešetkastom nosaču primijenimo metodu čvorova, vidi [22], za iznalaženje nepoznatih veličina (u našem slučaju sila u štapnim elementima), moguće je ispisati jednadžbe ravnoteže za svaki čvor. Računanje nepoznanica svodi se na rješavanje sustava linearnih jednadžbi, odnosno za naš navedeni primjer potrebno je riješiti sustav sedam jednadžbi sa sedam nepoznanica. Opterećenja u različitim čvorovima možemo prikazati vektorom $b$. Ovisno o broju nepoznanica, odnosno o veličini matrice $A$, možemo primijeniti različite metode za rješavanje. Najjednostavnija metoda za rješavanje sustava linearnih jednadžbi je Gaussova metoda eliminacije koja se interpretira kao faktorizacija matrice sustava $A$ na produkt trokutastih matrica. Pomoću računala moguće je brzo i točno riješiti sve veće i veće sustave što inženjerima omogućuje rješavanje sve složenijih problema. U primjeru sa Slike 1., opterećenja u različitim čvorovima daju različite vektore $b \mathrm{u}$ sustavu linearnih jednadžbi $A x=b, \mathrm{~s}$ istom matricom sustava $A$. Za slučaj velike matrice $A$ te kako bi se smanjilo vrijeme računanja i izbjeglo ponavljanje koraka Gaussove eliminacije na matrici A, pri rješavanju sustava za svaki različiti vektor $b$, moguće je napraviti $L U$ faktorizaciju matrice $A$. Također, ranije u radu, opisani problem vlastitih vrijednosti i 
spektralna dekompozicija nalaze praktičnu primjenu u dinamici konstrukcija, posebice u slučajevima iznalaženja odziva konstrukcije izložene slučajnim procesima. Primjer može biti ponašanje više etažne zgrade u slučaju djelovanja potresa. U tom slučaju rješavanje problema svodi se na modalnu seizmičku analizu, odnosno dinamičku analizu linearnih sustava sa $n$ stupnjeva slobode zasnovanoj na metodi razvijanja po vlastitim oblicima, ili kako se još naziva analizi po tonovima ili modalnoj analizi, vidi [20]. Nadalje, tipičan inženjerski primjer je vibriranje konzole kojoj je dana početna defleksija i potom je puštena da slobodno vibrira. Njezino vibriranje je problem vlastitih vrijednosti, gdje su vlastite vrijednosti prirodne frekvencije konzolne grede, a vlastiti vektori njeni modalni oblici.

\section{Zaključak}

Ovaj rad daje pregled samo nekih matričnih faktorizacija, onih koje se često primjenjuju. Osnovna ideja rastava ili faktorizacije matrice je njen zapis kao produkt jednostavnijih matrica čime se ubrzava rješavanje raznih problema kao što su problemi određivanja vlastitih vrijednosti, rješavanja sustava linearnih jednadžbi i rješavanja linearnog problema najmanjih kvadrata. Osvrt na bilo koju od faktorizacija ostavlja dojam jednostavnosti, no samo jednostavnosti u teoriji, ne i u računanju, a naročito ne u računanju matrica većih dimenzija. Primjerice, sustava linearnih jednadžbi oblika $A x=b$, pri čemu je $A$ kvadratna $n \times n$ regularna matica, a $b n$ dimenzionalni vektor, naoko je jednostavan za rješavanje, naročito za mali broj jednadžbi i nepoznanica. Sustavi linearnih jednadžbi imaju široku primjenu, a to su obrada digitalnih signala, aproksimacija nelinearnih problema u numeričkoj matematici i različite procjene i predviđanja, u fizici, kemiji, računarstvu i ekonomiji. Primjerice, LU faktorizacija i QR faktorizacija naročito su korisne u slučajevima kad se rješava više sustava linearnih jednadžbi s istom matricom $A$. Rješavanje sustava ipak krije mnoge zamke iako nam za njihovo rješavanje stoje na raspolaganju moćna računala. Može se samo spomenuti neke od problema, kao što je problem točnosti ulaznih podataka, problem smještanja podataka u računalo, dakle memorijskog prostora, broj operacija vezanih za odabranu metodu računanja, te aritmetika računala i točnost izlaznih podataka. Za rješavanje spomenutog sustava razvijaju se brojne iterativne metode, vidi [2], kod kojih se iteriranjem pokušava poboljšati neka početna aproksimacija, tako da u svakoj iteraciji greška bude što manja. Nadalje, primjenom odgovarajućeg kriterija zaustavljanja nekog iterativnog procesa postiže se dovoljno dobra aproksimacija za traženi vektor. Jordanova forma i Schurova dekompozicija daju bitne informacije o matrici koje se često koriste kod analize numeričkih algoritama. Schurova dekompozicija korisna je u 
numeričkom računanju jer unitarnom sličnošću matricu prevode u jednostavniji oblik. Ovaj rad nije posvećen numeričkom testiranju iteracija spomenutih metoda, vidi [4], [12], [17], već daje pregled matričnih faktorizacija i mogućnosti izračunavanja traženih podataka, ovisno o klasi matica, njihovoj formi i određenim svojstvima.

\section{Literatura}

[1] Atkinson, K.E. , An Introduction to Numerical Analysis, John Wiley and Sons, New York, 1989.

[2] Björck, W., Dahlquist, G, Numerical Mathematics and Scientific Computation, SIAM, Philadelphia, 1999.

[3] Bosner, N., Fast Methods for Large Scale Singular Value Decomposition, Doktorski rad, Matematički odjel PMF-a, Zagreb, 2008.

[4] Datta, B.N., Numerical Linear Algebra and Applications, SIAM, Philadelphia, 2010.

[5] Demmel, J.W., Applied Numerical Linear Algebra, SIAM, Philadelphia, 1997.

[6] Drmač, Z., Numerička analiza I - predavanja, Matematički odjel PMF-a, Zagreb, 2008.

[7] Drmač, Z., Hari, V., Marušić, M., Rogina, M., Singer, S., Singer, S., Numerička analiza, Matematički odjel PMF-a, Zagreb, 2003.

[8] Golub, G.H., Van Loan, C.F., Matrix Computations, The Johns Hopkins University Press, Baltimore-London, 1993.

[9] Horn, R.A., Johnson, C.R., Matrix Analysis, Cambridge University Press, Cambridge, 1990.

[10] Horn, R.A., Johnson, C.R., Topics in Matrix Analysis, Cambridge University Press, Cambridge, 1991.

[11] Horvatić, K., Linearna algebra, Matematički odjel PMF-a, Zagreb, 1995.

[12] Moler, C.B., Numerical Computing with MATLAB, SIAM, Philadelphia, 2004.

[13] Rogina, M., Singer, S., Singer, S., Numerička matematika - predavanja, Matematički odjel PMF-a, Zagreb, 2008.

[14] Parlett, B.N., The Symmetric Eigenvalue Problem, SIAM, Philadelphia, 1998.

[15] Radošević, I., Jacobijeve metode za računanje singularne dekompozicije matrice, Ur. Arbanas, Ž., Zbornik radova KNJIGA XVII , 247-269, Građevinski fakultet u Rijeci, Rijeka, 2014.

[16] Stoer, J., Bulirsch, R., Introduction to Numerical Analysis, Springer-Verlag, New York, 2002.

[17] Strang, G., Linear Algebra and It's Applications, Thomson, Belmont, 2006.

[18] Trefethen, L.N., Bau, D., Numerical Linear Algebra, SIAM, Philadelphia, 1997.

[19] Zhang, F., Matrix Theory, Springer-Verlag, New York, 1999.

[20] Čaušević, M., Dinamika konstrukcija - diskretni sustavi, ŠK, Zagreb, 2005.

[21] Kreyszig, E., Advanced Engineering Mathematics, $9^{\text {th }}$ edition, John Wiley and Sons, New York, 2006.

[22] Simović, V., Građevinska statika I, Građevinski institut, Zagreb, 1988. 